\title{
Tangence
}

\section{Liminaire. Face à l'extrême, les lieux de la critique}

\section{Michael Rinn, Philippe Mesnard, Michael Rothberg, Emmanuelle Danblon, Jean-Paul Dufiet et Georges-Élia Sarfati}

Numéro 83, hiver 2007

L'extrême dans la littérature contemporaine. Le corpus de la Shoah en question

URI : https://id.erudit.org/iderudit/016762ar

DOI : https://doi.org/10.7202/016762ar

Aller au sommaire du numéro

Éditeur(s)

Tangence

ISSN

1189-4563 (imprimé)

1710-0305 (numérique)

Découvrir la revue

Citer ce document

Rinn, M., Mesnard, P., Rothberg, M., Danblon, E., Dufiet, J.-P. \& Sarfati, G.-É.

(2007). Liminaire. Face à l'extrême, les lieux de la critique. Tangence, (83), 5-23. https://doi.org/10.7202/016762ar 


\section{Liminaire}

\section{Face à l'extrême,}

\section{les lieux de la critique}

Débat animé par Michael Rinn, avec la participation de Philippe Mesnard, Michael Rothberg, Emmanuelle Danblon, Jean Paul Dufiet et Georges-Élia Sarfati.

\section{Michael Rinn}

Ces dernières années, les sciences humaines ont considérablement renouvelé leurs recherches consacrées au corpus de la Shoah. Par delà le cadre institutionnel du domaine scientifique, ces recherches ont contribué à l'établissement et à la validation d'un vaste consensus social sur la nécessité du combat contre le racisme, sur le bon usage de la mémoire historique et sur l'engagement de la responsabilité individuelle face aux nouvelles formes de barbarie, sorte de Nouvel Organon dont il faudra mesurer la portée idéologique. En interrogeant les modèles théoriques, notre débat cherchera à dresser un état des lieux de nos différentes disciplines littéraires. Afin de contribuer à la Kulturkritik contemporaine, l'entretien portera sur trois concepts qui définissent largement la place que nos sociétés actuelles accordent à la représentation de la Shoah: l'esthétique, l'éthique et la politique.

\section{L'esthétique}

Même s'il y a de fortes raisons de penser que la problématique de l'extrême se situe à l'intersection de ces trois concepts, je suggère d'ouvrir le débat avec l'esthétique. Évoquant une rupture de civilisation provoquée par l'extermination - la cassure d'Auschwitz nombreux sont les commentateurs qui remettent en question la définition traditionnelle du genre du discours. Aussi faudrait-il lever, du moins partiellement, l'opposition entre fiction et nonfiction, littérature et témoignage, modèle de pensée vraisemblable et parole véridique. Or, comment appréhender la rencontre de ces notions, rencontres qualifiées d'improbables, voire de non pertinentes avant Auschwitz? Par ailleurs, la conception du témoignage comme nouvelle forme littéraire parait problématique. S'il est vrai que toute représentation d'une réalité historique ou d'un vécu 
personnel appelle à une esthétisation langagière, aussi épurée soitelle, le témoignage perd inévitablement de sa véracité pour gagner en crédibilité. Sa valeur esthétique répondrait à sa fonction communicative, qui consiste à se faire comprendre pour combattre ensemble l'oubli. D'un point de vue méthodologique, cette approche invite à la critique des instances énonciatives et des différentes procédures de référentialisation à l'œuvre dans le texte. L'enjeu de la réflexion porte ainsi sur la modélisation nouvelle de l'espace littéraire, mais également sur la formalisation de la pensée sociale. Enfin, à l'instar du succès réservé aux Bienveillantes de Jonathan Littell à la rentrée littéraire française de 2006, il faut s'interroger sur la fin proclamée du topos de l'irreprésentable, levant les interdits de figuration de jadis. L'extermination aurait conduit à la destruction progressive des normes esthétiques; mais comment définir les effets de l'art, dorénavant conventionnels, pour éviter la jonction dangereuse entre «tout peut être dit» et «tout se vaut»?

\section{L'éthique}

Venons-en à présent au concept d'éthique. Envisagée selon la tradition comme "un ensemble de règles qui régissent la vie en société », l'éthique a été radicalement remise en question, voire évacuée, par l'expérience de l'extrême. Pour éviter les pièges de l'oubli ou de l'indistinction qui favorisent la montée de la barbarie, nos sociétés de l'après-Auschwitz ont proclamé un nouvel impératif catégorique - Plus jamais ça! - dont le garant moral serait le témoin-survivant. Même si l'appel lancinant est resté sans lendemains, force est de constater la charge symbolique toujours croissante que nos sociétés actuelles attribuent à la parole du témoin. On peut penser que ce dernier revêt le rôle d'un acteur social idéalisé dans un monde désenchanté, voire obscurci, par l'extrême; il assurerait la pérennité de la raison agissante, la transmission fidèle du passé et la lisibilité du présent. En somme, il permettrait à l'humanité de douter encore d'elle-même, pour qu'elle puisse réaffirmer ses principes éthiques. L'idéalisation du témoin appelle à la critique. D'une part, on peut supposer qu'à l'heure où les derniers survivants des camps nazis vont disparaître, nos sociétés souffriront de la perte de porteurs de sens historiques et civiques, et que leurs paroles risqueront d'être récupérées et manipulées par des démagogues. D'autre part, et sur un plan théorique, il faut s'interroger sur la conception éthique de la communication. La production et la diffusion de la parole des témoins nécessitent de la 
part du récepteur des capacités d'interprétation. On peut reconnaître ici l'urgence d'une modélisation éthique de la lisibilité, permettant de définir les procédures de calcul de sens et d'évaluation de la portée culturelle, esthétique et artistique de l'écriture de l'extrême. Cependant, cette revendication peut contribuer à la formulation d'une véritable déontologie interprétative qui, en raison de son dispositif consensuel, ne pourra échapper à des postulats normatifs, voire moralisateurs.

\section{La politique}

Pour finir, réfléchissons sur le concept de politique face à l'extrême. À cet égard, la critique dresse un bilan contradictoire. D'une part, nous sommes nombreux à récuser l'usage politique de la mémoire du génocide auquel réfère, de manière emblématique, l'ordre civique du "devoir de mémoire». Alors que les tenants et aboutissants de ce prétendu devoir sont loin d'être clairement établis, on s'accorde sur le fait que l'excès de commémoration institutionnelle est source d'ambiguittés: Auschwitz est devenu un lieu commun du monde occidental, servant de fonds argumentatif à une politique de la mémoire destinée à expier les sentiments de culpabilité collective, à identifier les «forces du mal» du passé et du présent, ainsi qu'à promouvoir l'idéologie de la responsabilité individuelle. On a l'impression que cette politique, à laquelle participe l'industrie culturelle, cache derrière le consensus mémoriel la face hideuse de la barbarie toujours agissante. D'autre part, certains auteurs et commentateurs des récits du génocide ne manquent pas de rappeler l'enjeu politique de ces derniers, leur attribuant une fonction cognitive d'alerte. Le lecteur de ces textes pourrait ainsi prendre conscience de la précarité de la paix sociale et de la fragilité de sa propre humanité. Enfin, l'analyse des structures narratives et des procédés rhétoriques qui caractérisent l'écriture de l'extrême, tels que l'usage des figures discursives de la véhémence comme l'ironie, le sarcasme ou l'injure, permet d'identifier une pratique de la subversion de l'ordre établi. Ainsi, la critique du corpus de la Shoah altère non seulement les concepts d'esthétique et d'éthique existants, mais conduit également à porter un regard différent sur la politique actuelle. 


\section{Philippe Mesnard}

Je voudrais prendre part à ce débat avec quelques remarques qui seront à la fois des préalables à mes propres réflexions et une manière de répondre dans son ensemble au texte qui nous interpelle.

La réalisation historique du double système concentrationnaire et génocidaire nazi viendrait nous signifier - ce serait comme sa leçon - qu'il est difficile de détacher l'esthétique de l'éthique, tout en concevant l'une et l'autre séparément, comme nous y invite Michael Rinn, et sans risque de confusion. Maintenir l'articulation entre esthétique et éthique est à mon sens une façon de prendre acte de ce qui a eu lieu, tel que cela s'est réalisé comme atteinte au lien même qui permet à l'humanité de tenir. Déjà là, il y a un engagement contre ce que le nazisme représente. En effet, le nazisme, par l'entreprise qu'il a menée en poussant au plus loin l'esthétisation du pouvoir jusqu'à le parer de tous les attributs de la puissance (et la guerre est ici le prolongement même de cette esthétisation en ce qu'elle n'est plus, à partir de la guerre d'Espagne, dissociable de sa propre représentation) et, conjointement, en poussant au plus loin la destruction des liens éthiques sur lesquels repose la possibilité même d'une communauté humaine, le nazisme, donc, accomplit cette rupture entre esthétique et éthique, il la consomme au prix de la destruction de l'éthique. Un exemple de cette destruction n'est pas seulement l'assassinat méthodique de groupes humains entiers, les Juifs les premiers, mais que cette violence sans répit ne peut s'exercer que derrière des simulacres dont les Sonderkommandos ont été un des cas les plus désespérants. On pourrait également donner, pour second exemple, cette société allemande qui, subissant la double terreur du régime et des bombardements, trouve la figure de sa destruction éthique dans le suicide de l'enfant sur lequel se termine Allemagne année zéro (1947) de Roberto Rossellini.

Une chose est que le rapport politique entre esthétisation de la représentation et puissance est anthropologiquement très ancien, une autre chose que l'esthétisation sans limite du nazisme soit strictement concomitante à la réalisation de la destruction même de l'humanité, de toute humanité, dans l'homme. Que l'on y regarde bien, il n'y a plus aucune place pour l'éthique. En revanche, l'esthétique se prolonge et s'accomplit en une apothéose de la destruction par excellence: le feu d'artifice des Katiouchas confine au sublime pour nombre de spectateurs. Les torches 
urbaines, dont le feu est venu du ciel, viennent répondre, de manière spectaculaire, à l'invisibilité du génocide des Juifs dont l'horreur, après s'être déroulée sous terre, s'est élevée en fumées incolores zum Himmel, comme on disait alors. Du ciel... et retour vers le ciel - un ciel vide et, surtout, retourné (grave affaire pour la théologie), dont il ne pouvait plus pleuvoir que du sang (Rawicz).

Aussi, me semble-t-il, s'il y a un engagement que je me donne d'assumer, en tant que chercheur, et qui pour moi est indissociable de ma recherche, c'est bien de réinscrire l'éthique dans l'esthétique, de forcer l'esthétique pour y dégager un espace, fut-ce celui d'un non-lieu, où se déploie la question éthique. Et cette question, les œuvres testimoniales, en premier celles des survivants, l'introduisent et la portent. Comment penser l'éthique après la destruction de l'éthique, à partir du vide laissé au lieu même de l'articulation humaine - sans faire de la question éthique une voie pour sacraliser le vide (celui de la dévastation comme celui de la réification) qui résulte de cette histoire, et sans ouvrir non plus à quelque mystique du désastre? Tel serait bien le pari, l'engagement - l'engagement tient toujours à un pari et relève du choix et du risque, de l'acceptation, voire de l'exposition à la perte aussi —, tel serait donc bien le pari à relever pour le chercheur, lequel, à mes yeux, ne doit jamais être dissocié du pédagogue. Deux remarques, cependant, concernant l'esthétique et le temps.

L'esthétique. Il ne s'agit pas là de produire un discours antiesthétique, comme un remake de sentences iconoclastes, ce serait aussi ridicule que l'attitude de ceux qui voudraient jouer la rationalité comme «le» pathos: l'enjeu se porte sur l'articulation et la mise en tension des deux et l'espace intervallaire qui s'ouvre alors. La question est précisément de rétablir une économie entre éthique et esthétique quand l'esthétique a envahi le monde et que l'éthique en a été expulsée. Au risque d'être elliptique, je pense que la «réussite» de l'esthétique tient moins aux représentations, au «spectacle» comme tel, qu'à la prodigieuse accélération du temps de leur perception (cette accélération, le quotidien y est tout entier assujetti, tous les sens y sont aliénés: l'accélération des plans et des montages cinématographiques comme l'augmentation des rythmes musicaux en sont des exemples parmi les plus flagrants).

Le temps, donc. Nous vivons dans un monde de représentations que l'on ne saisit que par l'autoreprésentation du monde même. De là, l'écrasement de toute distance et le fait que ce monde 
du spectacle soit déterminé par le rapport temporel qui l'institue, un rapport qui se développe sur le mode exclusif de l'urgence, de l'instantané, de l'écrasement du temps historique (lequel nécessite un déploiement spécifique au regard duquel la vitesse de l'actualité ne laisse plus d'espace, ni central, ni même marginal). C'est pourquoi saisir l'histoire et y prendre position demandent de se dégager d'une actualité également envahie par la vitesse de l'information et monopolisée par elle. Dissocier la contemporanéité de l'événement de son actualité - et enseigner cette dissociation - est peut-être le premier geste éthique qui ait pleinement valeur d'engagement, dans la mesure où il rétablit précisément une mesure de l'histoire: l'histoire comme mesure et non comme fin, apocalypse, terreur, fulguration, eschatologie. Au commencement, il devra désormais y avoir cette séparation du temps dans le temps qui le détache de l'immédiateté (l'immédiateté: l'abolition de toute médiation, de tout lien symbolique et du symbolique même en tant qu'il régule les rapports de l'imaginaire au réel et inversement; l'immédiateté: l'apogée du pathos aux dépens de toute raison). Sans l'opération de cette séparation, de ce détachement, on stationne dans l'illusion de savoir, dans l'illusion réflexive d'un sujet qui se prend pour celui qui sait tout tout de suite. Cette ellipse me ramène à du concret, au concret du chercheur: face aux crimes, c'est donc la distance que je préconise, distance qui reste la façon de ne pas être entraîné par le torrent de leur actualité, ou du moins d'y flotter.

Un dernier mot pour dire que, s'il y a de l'extrême, pour nous, chercheurs, celui-ci se situe, me semble-t-il, au lieu de ce domaine de recherche où ce que la mémoire a de plus normatif est en tension permanente avec ce que la violence a de plus radical. Là, se trouveraient des enjeux de pensée particulièrement critiques et exigeants pour notre âge - une de ces exigences étant de maintenir la tension entre mémoire et violence comme un questionnement.

\section{Michael Rothberg ${ }^{1}$}

Il est, semble-t-il, juste de commencer avec la question esthétique. Bien que les implications éthiques et politiques de la Shoah soient vraisemblablement les plus pressantes, elles sont immanquablement traversées par la question de la représentation autour de laquelle elles s'articulent. Depuis le début, la question de l'Holocauste s'est accompagnée de nombreux débats autoréflexifs

1. Section traduite par Audrey Évrard. 
sur l'art et la dimension esthétique — que ce soit dans le discours philosophique (on peut noter ici l'intervention d'Adorno sur «la poésie après Auschwitz») ou directement dans les pratiques esthétiques (ainsi, la poésie de ghetto autocritique d'Abraham Sutzkever). Alors que les premiers débats concernant l'esthétique de l'Holocauste tendaient le plus souvent vers un discours moral et moralisateur - ce qui peut être représenté, ce qui devrait ou ne devrait pas être représenté —, aujourd'hui, la problématique des limites se retrouve saturée par la prolifération des modes de représentation. Une telle évolution ne signifie pas nécessairement que toutes les représentations de la Shoah soient devenues systématiquement acceptables ou tout aussi valides. Il est au contraire préférable de concevoir le domaine esthétique comme un champ de contestation, une aire où s'affrontent diverses positions quant aux significations et aux conséquences de l'extrême. Au lieu du problème des «limites de la représentation", nous sommes aujourd'hui confrontés au problème de la «disposition des représentations» - les liens entre différents modes de représentation, les relations de force entre différentes mémoires dans une sphère publique hétérogène, elle-même définie par une multiplicité d'histoires de l'extrême (non seulement la Shoah, mais aussi le colonialisme, l'esclavage, les génocides contemporains, le terrorisme d'état et nonétatique). Bien que l'éthique et la politique s'articulent toujours par l'intermédiaire de l'esthétique, les questions esthétiques sont aujourd'hui d'emblée éthiques et politiques.

La raison d'être du témoignage est motivée par plusieurs impératifs différents, voire contradictoires. Parmi ceux-ci, je distinguerais notamment sa fonction pédagogique. Le témoignage du survivant peut apporter un enseignement utile aux générations futures dans l'établissement d'une relation éthique à l'extrême. Les témoignages autoréflexifs, comme ceux par exemple de Charlotte Delbo ou de Ruth Klüger, produisent une réponse ambivalente appropriée: ils interpellent les lecteurs dans leur rôle de témoins secondaires tout en marquant la distance qui sépare tout survivant de l'extrême des non-survivants. Cette double stratégie a pour but d'instaurer un équilibre fragile - ce que Dominick LaCapra appelle «trouble empathique» (empathic unsettlement). Une telle stratégie ne dépend pas d'une idéalisation du survivant; je crois, en effet, qu'elle peut activement militer contre une telle idéalisation. La difficulté qu'il y a à vouloir établir une position stable vis-à-vis de tels témoignages - parce qu'ils nous attirent et nous repoussent à la fois - freine et décourage toute inclination à «fixer» la place du 
survivant, que ce soit par l'idéalisation ou l'abjection. Le mode esthétique que j'appelle «réalisme traumatique» est éthique dans la mesure où il constitue une tentative de déstabiliser la relation entre les survivants et les générations futures, sans pour autant effacer la rupture radicale que représente l'expérience de l'extrême.

Enfin, il est clair que le nouvel impératif catégorique qui a été attribué au fait de la Shoah — «plus jamais!» — n'a pas eu l'impact souhaité en empêchant de nouveaux génocides et d'autres formes de manifestation de l'extrême dans le monde après l'Holocauste (à moins que cet impératif ne soit à interpréter que dans son sens le plus étroit, à savoir la prévention de politiques génocidaires à l'encontre du peuple juif). De plus, on peut facilement trouver des exemples de politisation abusive de l'Holocauste partout dans le monde, et ce des deux côtés des luttes politiques. Je reste néanmoins optimiste, même si je garde quelques réserves vis-à-vis des politiques mises en place pour préserver la mémoire de l'Holocauste. Les répercussions politiques des concepts de mémoire et de représentation sont quasiment impossibles à prévoir. Je suggère donc, qu'au lieu de pointer du doigt tous les cas où l'invocation du génocide nazi a échoué dans sa fonction de mettre fin au fascisme, aux génocides ou au "nettoyage ethnique», nous prenions en considération les réactions extrêmement élaborées qui ont suivi la Shoah — dans la littérature et dans les arts, dans le discours philosophique et dans la théorie littéraire, la psychanalyse et l'histoire et que nous y puisions les éléments permettant de formuler une pensée et une action. Si nous acceptons de changer d'optique, en quittant notre posture cynique pour afficher un espoir prudent, nous verrons d'abord comment, partout dans le monde, plusieurs communautés menacées ont su tirer un enseignement de la Shoah et de ses conséquences. Mais nous reconnaîtrons également que cette réaction à la Shoah s'est construite sur, et a tiré, des leçons d'autres histoires qui l'entourent et viennent butter contre elle dans la sphère publique; c'est ce à quoi je fais référence dans mes travaux récents, lorsque je parle de la «multidirectionalité» de la mémoire. Cette interaction interculturelle et transnationale a été largement gommée et masquée en raison notamment des notions préconçues que certaines communautés (les Juifs, les Noirs, etc.) ont sur la "propriété» de l'histoire, de la mémoire et de l'expérience. Il est nécessaire qu'une nouvelle politique mémorielle de la Shoah soit mise en place et qu'elle se fonde sur le principe que les histoires de l'extrême n'appartiennent à personne en particulier - et qu'elles sont donc accessibles à tous. 


\section{Emmanuelle Danblon}

Les trois modes d'appréhension de la question (esthétique, éthique, politique) convergent vers une question qui a trait au concept de "post-humanité». Ce concept peut servir de paradigme pour la description d'une nouvelle doxa produite après Auschwitz et qui aurait comme caractéristique essentielle de réduire les catégories, de brouiller les frontières. Les conséquences de cette réduction opèrent aux trois niveaux de notre réflexion:

- esthétique : brouillage de la frontière entre fiction et réalité (d'où le statut problématique du témoignage);

- éthique : brouillage de la frontière entre bourreaux et victimes. Ici, le cas du roman de Littel me paraît exemplaire des conséquences doxiques du brouillage. Sous prétexte de dire que le "héros» du roman est, somme toute, un homme ordinaire, on décrit en réalité un personnage qui a tous les traits de la perversité (y compris sexuelle) et on donne implicitement à la perversité (une marge de l'humanité) le statut de norme;

- politique: brouillage de la fonctionnalité des discours (et des genres) dans la vie politique que révèle l'expression «devoir de mémoire». Dans un système aristotélicien classique, il y a des moments où la cité commémore sans critiquer (c'est le genre épidictique) et des moments où elle critique sans commémorer (judiciaire et délibératif). Ces distinctions se basent sur la distinction esthétique entre fiction et réalité qui est elle-même brouillée.

L'ensemble de ces réductions a pour point commun la mise à distance du paradigme de la modernité symbolisée par les Lumières et sa conception de la raison basée, entre autres, sur l'idée même de distinction (la capacité à discriminer est à la base de l'activité critique à l'œuvre dans tous les domaines d'une société ouverte). La postmodernité basée sur la doxa implicite de la posthumanité érige l'indistinction en nouvelle norme, ce qui débouche (consciemment ou non) sur une lecture perverse du monde et de l'humanité. Voici d'ailleurs ce que seraient ces traits de la perversité:

- réduction du symbolique au littéral;

— haine de soi (Littel);

— volonté de "tout dire» comme réponse aux délicates questions de l'ineffable; 
- destruction du sens;

- etc. (la liste n'est pas exhaustive).

Le livre pénétrant de Bernard Williams, Vérité et véracité, retrace le traitement de ces deux concepts dans la modernité et montre qu'une partie de la défiance envers la vérité pourrait être éclairée par notre problématique. Dans le cadre de toutes ces réductions, le concept de vérité vole en éclats. Williams parle de «subversion», que l'on pourrait rapprocher de cette connexion objective entre la posthumanité et les traits de la perversité décrits plus haut.

Une autre partie de la doxa contemporaine met de l'avant l'importance de la mise en récit d'événements traumatiques par un besoin de donner du sens et se trouve parfois également en butte avec le problème de la vérité. D'où les délicates questions touchant à la "vérité du témoin ». La société contemporaine a ressenti le besoin de redonner droit de cité à la narration, sans trop savoir quel statut lui donner: doit-elle remplacer la vérité?

Une proposition pour l'époque contemporaine: plutôt que de jouer la vérité contre la véracité, Williams propose de les réconcilier. Il dit explicitement que cette question concerne directement la question de la Shoah, sans pour autant l'approfondir ${ }^{2}$. Il y a, à mon sens, une piste possible pour cette réconciliation dont l'épistémologie poppérienne ouvre la voie en proposant de séparer, dans la raison, le moment de la découverte de celui de la justification. Le moment de découverte correspond à celui qui est valorisé dans la mise en récit, qui permet de donner $d u$ sens. À ce moment, la question de la vérité est suspendue, neutralisée. Vient ensuite le moment de la justification (comment traite-t-on la représentation à laquelle le récit donne lieu en fonction de l'institution à laquelle elle s'adresse?). À ce moment, le concept de vérité redevient opérant et les mécanismes de la critique peuvent fonctionner. Cette conception en deux temps de la rationalité permet de ne pas faire porter tout le poids de la raison sur la narration et, partant, sur la figure symbolique du témoin. Une fois les récits enregistrés, ils pourront encore faire l'objet d'un travail critique et de justifications, et ce même après la mort de tous les témoins, sans pour autant que cela soit ressenti comme un sacrilège.

2. Bernard Arthur Owen Williams, Vérité et véracité. Essai de généalogie [2002 en anglais], traduit par Jean Lelaidier, Paris, Gallimard, coll. «NRF essais », 2006, p. 249. 
La pensée mythique (qui était en partie obscurantiste) utilisait prioritairement la fonction narrative (ce que Carlo Ginzburg appelle le "paradigme indiciaire»). La pensée moderne a écrasé ce paradigme et a voulu le remplacer par une conception désincarnée de la raison dont la "vérité» était devenue le dogme. La pensée contemporaine, qui refuse la voie potentiellement perverse de la postmodernité (laquelle se réconcilie de fait avec le caractère obscurantiste de la pensée prémoderne), peut trouver une piste de résolution dans la réconciliation par articulation des deux paradigmes: la narration puis l'argumentation, donner du sens puis critiquer ce sens. Séparer pour réarticuler la découverte et la justification.

\section{Jean-Paul Dufiet}

À partir des années 1980, en Europe, et plus particulièrement en France, après une longue période au cours de laquelle elle connut, pour de nombreuses raisons, l'indifférence et la minimisation, la Shoah a provoqué une inflation discursive. Une sorte de discours aux accents de shoah-centrisme a comme menacé l'événement lui-même; elle a été dévoyée parce qu'elle est devenue la maîtresse référence. Du coup, les réflexions les plus sérieuses, et les plus nécessaires, ont côtoyé des dérives indéfendables: la Shoah est devenue une commodité de langage, un topos dans la communication médiatique, culturelle et politique. Au plan politicointellectuel, on a assisté à une réinterprétation génocidaire systématique de l'histoire; au plan éthique, être la victime d'un génocide est entré dans le domaine des revendications identitaires; au plan esthétique, la Shoah est devenue le contexte scabreux et sulfureux de nombreuses fictions kitsch destinées à donner le frisson du mal absolu. Ainsi la Shoah s'est-elle retrouvée, en même temps, glosée et suffoquée.

Toutefois, il convient de dépasser ce moment, sans doute inévitable, car, quelles que soient ces dérives incontrôlables, il reste incontestable que le devenir de l'Occident ne peut se penser hors de l'ombre de la Shoah. Elle domine, et dominera probablement encore pendant longtemps, le destin et les enjeux de l'Occident. Pourquoi ? Pourquoi la Shoah réside-t-elle dans le filigrane du devenir de l'Occident? Ou, en d'autres termes, de quelle manière la Shoah est-elle toujours là, en compagnie du destin de l'Occident? Ce n'est probablement pas parce qu'elle pourrait se répéter à l'identique. Chacun sait que l'histoire est rusée, et qu'il faut 
toujours être plus attentif à ses métamorphoses et à ses mutations qu'à ses répliques. Si la Shoah barre les chemins de la politique, de la pensée et de l'art, ce n'est pas non plus en raison de l'incontestable degré d'horreur qu'elle a atteint. Certes, l'idée qu'un niveau absolu et indépassable de l'abomination a pu être atteint laisse médusé. Mais, essentiellement, la Shoah continue de se représenter au futur de l'Europe, parce que cet événement, en dépit de toutes les utiles explications historiques, politiques, éthiques et culturelles qu'il a suscitées, demeure toujours, pour la conscience occidentale, un point d'interrogation. Et il y a bien quelque soupçon qu'il le demeurera. C'est en ce sens que l'extrême demeure. C'est bien dans la mesure même où elle continue de faire question que la Shoah se présente à l'avenir de l'Occident. Et il n'est sans doute pas erroné de dire que les autres génocides de l'histoire, occidentaux ou pas, que nul ne cherche à nier, prennent, au contraire de la Shoah, une place dans le sens humain. Même si c'est dans les errements des civilisations, les autres génocides sont compréhensibles. Aussi effrayants qu'ils soient, ils font sens sur le plan historique, politique ou anthropologique. La Shoah, en revanche, demeure cette "folie rationnelle", comme dit Primo Levi, qui donna au SS impassible le seul et continu spectacle de la mort illimitée. Et toutes les motivations, toutes les descriptions rigoureuses des étapes et des mécanismes, ainsi que toutes les causalités logiques qui ont mis en œuvre l'extermination demeurent insuffisantes, pour ne pas dire impuissantes, à rendre la Shoah assimilable au sens. Elle est donc exactement cette unicité-là, irréductible. Et sans doute, ce sens manquant de la Shoah est-il la source de la sacralité noire, si déplorable et si scandaleuse, dont on l'entoure parfois. La Shoah est donc une aporie du sens qui repropose son point d'interrogation à chaque pas décisif de l'Europe, qu'il soit politico-éthique ou artistique.

Au plan politico-éthique, la Shoah explique pour une bonne part le projet de construction de l'Europe: la protection des droits des minorités, l'interdiction des discriminations de toute nature, l'attention minutieuse à toutes les victimes potentielles. La Communauté européenne n'a pas été seulement un instrument politico-militaire pour se défendre contre le communisme soviétique; les pays de la Communauté européenne ont aussi exercé, et continuent d'exercer, un contrôle politico-éthique des uns sur les autres. Comme si chaque pays, aussi civilisé soit-il, en raison du point d'interrogation de la Shoah, doutait de lui-même. Chaque nation européenne se protège d'elle-même, grâce aux autres. 
Dans le domaine esthétique, la Shoah a bouleversé l'une des questions centrales qui fondaient l'esthétique depuis les tragiques et les épiques grecs: il s'agit, bien sûr, de la représentation de l'horreur et de la monstruosité. Rappelons que la culture occidentale est née au cœur même de ces questions: intègre-t-on l'horreur dans l'art? Comment la montre-t-on, et à quelles fins? Le camp d'extermination a mis au cœur du continent occidental une horreur inassimilable, à laquelle nulle finalité dans l'ordre du sens humain ne peut être attribuée, puisqu'elle n'est que la présentation de la mort elle-même. La tragédie, que les Grecs nous ont léguée, est, on le sait, une forme de dialogue de l'homme avec le monstrueux divin; elle est le dernier moment de la déperdition de l'homme que l'on puisse montrer. Mais cette forme exprime aussi, et surtout, les droits et les sentiments de justice et d'injustice des antagonistes. L'extrême de la chambre à gaz et du four crématoire n'entre pas dans cette logique, ni dans aucune autre. Anéantissement et extermination: seuls les SS, dont la casquette porte une tête de mort, peuvent, sans s'effondrer, se représenter un tel monceau de cadavres.

L'extrême demeure, non pas parce qu'il est advenu dans l'histoire et qu'il fait tache dans la mémoire, mais parce qu'une part de l'extrême même échappe à la signification humaine.

\section{Georges-Élia Sarfati}

\section{L'esthétique}

On peut d'abord contester la préséance donnée ici à l'esthétique, bien qu'elle soit, je n'en doute pas, le résultat d'une décision de pure méthode. Ce primat peut en effet laisser craindre une propension première à l'esthétisation. Surtout entre "gens de lettres »... Autre préalable, qui ne définit pas seulement une précaution oratoire, et qui, pour nous, se traduit en impératif: l'indispensable levée des présupposés, à tout le moins de certains parmi les plus prégnants. S'agissant d'un sujet aussi sérieux, il convient d'éviter au mieux de ne pas être la dupe des «manières de dire» et, tout autant, des inférences que celles-ci autorisent. Mauvaises manières contre lesquelles nul ne saurait jamais assez se prémunir! La perspective scripturaire que l'on adopte sur le phénomène nazi ne cesse, en effet, de donner lieu à d'innombrables «contributions», notamment «savantes». Mais, sciemment, je ne veux pas ouvrir la littérature à ce moment de l'histoire, 
car, après ce choc essentiel, tout, précisément, n'est-il pas littérature?

Malgré tout, à moins de céder à la facilité, ou à une formidable présomption d'orgueil, la revendication acritique des normes de la civilisation retentit aujourd'hui comme une offense. Je ne pense pas que ce qui s'est dit et écrit, se dit et s'écrit ou se dira et s'écrira encore à propos d' "Auschwitz» puisse aujourd'hui s'incorporer à la littérature. Cette facilité nous porterait à trop vite "assimiler» l'avènement de l'inhumain en nous empressant de donner libre cours à l'ordre habituel, et habituellement reconduit des choses... La littérature, surtout contemporaine, a fui le référent, et s'est notamment ingéniée à se parfaire dans l'idiosyncrasie. Or, l'immense matériau scripturaire suscité par le phénomène nazi voit le retour massif du référent. Les scripteurs, une fois encore, sont happés par un objet qui n'est pas l'objet littéraire traditionnel, futil remanié. Fut-elle de l'ordre d'un néonaturalisme, l'exploration de cela défie le canon littéraire. Aussi, je ne suis pas de ceux qui professent que l'expression esthétique nous soit interdite après "Auschwitz»; en revanche, je sais, en tant que poète, qu' "Auschwitz» met en crise l'idée même de littérature. Un inestimable séisme au cœur de la mimèsis s'est produit à ce tournant de l'histoire, qui s'accompagne de la possible défaite du contrat de lecture bien normé que le scripteur témoin ne peut recomposer sans mal. De surcroît, s'il le fait, ce sera au nom d'un pari du type: lisez et peut-être croirez-vous, parce que moi-même, en dépit du fait que cela me soit arrivé, je ne peux pas encore l'imaginer, ni le faire entrer dans une représentation littéraire.

D’une part, tout scripteur, quel que soit ou quel qu'ait été son degré de proximité, ou d'implication, est obligé de faire fond sur cette impossibilité, à moins de pressentir d'emblée que le témoignage ne témoignera jamais que partiellement, au risque de manquer son objet; pour la simple raison que son dit est projeté dans un univers sémiotique radicalement hétérogène à cela même qui l'a suscité. Que dire alors du "lecteur» destiné qui ne s'égalera jamais à la narration qui se dévoile, et qui demeure, à cause de son extériorité, un faux-semblant sans contrepartie?

D'autre part, en quoi "Auschwitz» a-t-il été une "cassure», une "rupture de civilisation », et surtout pour qui? Pour ceux qui en ont subi le choc, et qui connaissent les terribles ondes de l'après-coup. Mais, pour les autres, il n'y a qu'absence de remords, honte cachée, ou tout simplement méconnaissance. Tout conspire 
donc à nous montrer le contraire: le cours compulsivement meurtrier de l'histoire, l'essor du négationnisme qui poursuit au plan du langage l'entreprise nazie d'annihilation, la reformation continuelle du prurit de haine d'un côté; de l'autre, la facticité d'une soi-disant civilisation qui a renoué, même artificiellement, avec son telos. Quant au nom d' "Auschwitz», il nous offre un cas très éloquent, proprement scandaleux, de réduction par la métonymie de l'immense entreprise d'anéantissement. J'en redoute pour ma part le pouvoir d'abstraction propre à désincarner le massacre et, par conséquent, à favoriser le relativisme autant que la mauvaise métaphysique.

\section{L'éthique}

Pardon de revenir sur ce point; mais s'agissant du travail de l'écriture face aux effets du phénomène nazi, j'éprouve beaucoup d'embarras à tenir pour acquis le distinguo éthique/esthétique. Sans doute les témoignages portent-ils, dans le meilleur des cas, l'éthique de leur esthétique, mais il faudrait affiner considérablement nos analyses pour échapper aux généralités. Peut-on échapper au piège de l'esthétisation sans commettre simultanément un immense manquement à l'éthique? Force est d'admettre qu'à travers les paroles du témoin, paroles médiatisées par le livre, ou paroles proférées face à un auditoire, pour autant qu'elles se supportent d'une visée éthique, celles-ci ne nous sont un tant soit peu communicables que parce qu'elles se supportent d'un ethos. C'est à cette dimension première que je reconnaîtrai d'abord l'éthique. Et toute parole proférée, écrite ou prononcée, se justifie à l'instant même de sa profération d'une forme qui est sinon la marque d'un choix, à tout le moins d'un dessein d'évidence pondéré par la situation. Par là même, cette profération, qu'elle articule ou non un témoignage audible — je veux dire qui atteint à la clairvoyance est d'emblée fondée sur la dictée d'une esthétique. Mais laquelle? S'agit-il des normes d'un genre déjà consacré, ou nouvellement institué par le fait même du témoignage? Ou bien cette esthétique doit-elle s'entendre, au premier chef, comme l'expression d'une aisthêsis, c'est-à-dire d'une sensibilité qui cherche, à travers formes et exigences éthiques, à se faire entendre? À ce point de l'analyse, c'est donc sous cet unique et très précis rapport de l'évaluation de ce qui a une valeur et qui est aux prises avec une sensibilité singulière que se joue le devenir du lien entre éthique et esthétique. D’où le nouveau primat qu'il me paraît nécessaire et urgent 
d'accorder à l'éthique, pour autant qu'en effet, ici, elle s'accorde à l'aisthêsis. Peut-on envisager ce lien consubstantiel autrement qu'à l'aune d'une distinction de méthode? Je ne le pense pas.

La question est alors de savoir si ce parti pris de dire peut s'accommoder d'un style solidaire, entendons par là une façon qui en appelle à l'éveil de l'autre, originairement étranger à l'attente du néant dont vient pourtant nous entretenir le témoin? Les "plus jamais ça» entendus dès après la Libération n'ont jamais eu valeur de décret pour personne, ni pour les victimes effectives, ni pour les victimes potentielles. Pareilles injonctions sont vaines, ce dont témoignent les multiples reprises, ainsi que, de ce point de vue, la vaine succession des générations conjuguée à la mobilité des préoccupations. Sans doute peut-on aussi discerner cette revendication endeuillée des identités comme une victoire posthume du national-socialisme, l'idéalisation du témoin me paraissant définir son point culminant. C'est le retour du référent évoqué tout à l'heure, mais d'un référent impossible, en l'absence duquel la personne du témoin, quand ce ne sont plus ses dits, tient désespérément lieu de substitut mortel. C'est avec cette dimension de l'absence que l'éthique contemporaine tente de compter depuis la seconde moitié du Xxe siècle. Après ceux que l'on nomme «les derniers témoins » (peut-être parce qu'ils étaient les derniers à s'autoriser la parole), nous nous demandons quelle fraction de l'humanité sera notre "référence», sachant que les derniers à parler défendaient, pensions-nous, l'accès à une vérité tangible qui tenait à leur seule présence. Ils étaient donc ceux dont le souvenir immergé dans ce que nous n'avons pas vécu s'interposait entre «nous» (ceux d'après «Auschwitz») et la déformation ou l'ignorance du "passé». Puis-je, pour l'avoir rencontré, fameux ou infâme, devenir à mon tour le témoin du témoin, et ainsi de suite ad infinitum? Puis-je, par suite, au mépris de toute sociologie, garantir par ma seule détermination à leur demeurer fidèle, m'assigner une mission testamentaire qui ferait lien entre l'inadvenu pour moi et l'après de leur disparition?

C'est pourtant, pour ne nommer qu'eux, ce que semble attendre Robert Antelme, et ce que Dionys Mascolo à son tour tente d'intenter auprès de la cohorte de lecteurs auxquels ce dernier se résout, quarante ans après, à exposer la lettre qu'il reçut du premier en $1945^{3}$. Nous entrevoyons ici que la question ouverte

3. Dionys Mascolo, Autour d'un effort de mémoire. Sur une lettre de Robert Antelme, Paris, Maurice Nadeau, 1987. 
de l'éthique laisse pendante la question lancinante de la pérennité du témoignage, de sa puissance d'impact ainsi que de la transmissibilité du statut de témoin. Témoin sans grandeur, témoin très inférieur au nombre toujours indénombrable de ceux qu'il faudrait encore "toucher », comme par une indicible grâce, à l'aide de la parole donnée et reçue. La question de l'esthétique, étroitement associée à celle de son enracinement dans l'ethos du témoin vivant, a trait, on s'en aperçoit, à celle de la recevabilité de son dit. Mais il faut aussi faire confiance à la déontologie immanente au langage; elle tient dans la mémoire lourde des mots, cette incoercible mémoire en laquelle les témoins eux-mêmes ont cru (fussentils passeurs de témoignages reçus): nous leur restons redevables de dire à notre tour plus et mieux que ce que nous pensons savoir. Le plus éclatant triomphe de cette mémoire des mots - même affectée par la tyrannie (l'enseignement de Klemperer dépasse de beaucoup la seule langue allemande) — n'est-il pas de contraindre les négateurs du crime à reconnaître, par le redoublement de l'affirmation propre au déni, cela même qu'ils veulent effacer?

Quoi qu'on en dise, cela aussi est à compter au nombre des effets du travail de l'écriture de la parole. L'idiome national, même saturé de contradictions, offre le spectacle significatif d'une lutte constante entre l'envers et l'avers du néant, une lutte qui se déroule par énoncés interposés. Énoncés envolés de l'archive. Faut-il alors douter du lecteur? Faut-il se montrer si craintif, si précautionneux à l'égard de « ses capacités d'interprétation »? En l'espèce, l'antidote à toutes les démissions ne me paraît pas seulement tenir dans l'"éducation à la lecture» mais, plus certainement, dans le couplage de deux apprentissages, garants ensemble de la vigilance, en place et lieu du «témoin vivant»: le sens critique aiguisé par la lecture et le jugement pratique aiguillé par la connaissance historique. Après les "derniers témoins", en matière de régulation éthique, il faut relever le défi d'un nouveau sens commun. Il serait fondé sur les normes d'une véritable culture populaire, assez riche pour se tendre à la moindre menace. C'est l'unique parade contre un nouveau déchaînement de la barbarie.

\section{Le politique}

À ce niveau de considération que, pour ma part, $\mathrm{j}$ 'aurais placé au premier plan, bien des questions interfèrent, de nature très différente. Est-il possible d'en démêler les enjeux? Rien de plus sujet à caution en effet que l'instrumentalisation du «devoir de 
mémoire", à travers réitérations, déplacements et recyclages qui vident cet impératif de tout esprit d'à propos. Primo Levi, soucieux de prolonger son témoignage par une injonction à la fois éthique et politique, a vu son exigence littéralement décontextualisée et peu à peu dévoyée. Cela nous indique, si besoin était, que depuis Machiavel une éthique de la justesse (exception faite du calcul) s'avère incompatible avec une politique authentiquement informée par le souci des sujets. Cette antienne, devenue le mot d'ordre de la décennie 1990-2000, a en effet servi de point de ralliement aussi bien à la concurrence des victimes qu'au recyclage des discours les plus dogmatiques, jusqu'au renversement de son sens ${ }^{4}$.

Par ailleurs, le fait qu' "Auschwitz» soit devenu le «lieu commun du monde occidental» atteste de ce que l'Occident n'est toujours pas revenu d'un ethnocentrisme invétéré. Et j'y vois, pour ma part, moins un pansement sur une blessure toujours béante que l'expression convoitée et combattue d'un judéocentrisme négatif. Comme si l'élimination de près de la moitié du peuple de l'Alliance pouvait valoir de rappel à un ordre éthique par ailleurs constamment récusé, si l'on en juge, justement, par l'histoire de la judéophobie occidentale. Aussi, ce n'est pas le moindre des paradoxes de l'extermination d'induire pour effet de discours la vaine tentative de recomposer du lien humain à partir du motif que l'Occident a tendanciellement cherché à gauchir, à escamoter jusqu'au point de chercher à l'éradiquer de manière systématique. N'oublions pas que, dans l'Europe des années 1939 à 1945, la chasse aux Juifs fait partie de la culture ambiante. Et voici que la Shoah sanctionne le terme de ce processus de néantisation symbolique par le passage à l'acte massif, «pur» et «simple». Ceci, on l'oublie trop souvent, résulte d'une concaténation de mécanismes à soubassements éthiques: éthique du racisme doctrinaire ou de la lâcheté ordinaire. L'épreuve de la banalité du mal demeure à cet égard emblématique de "ce que peut l'homme» et la tardive célébration des "Justes" retentit comme un terrible réquisitoire à l'égard du plus grand nombre, complices zélés ou panurges d'une majorité silencieuse. Alors, prendre au sérieux la conjonction du politique et de l'éthique après la Shoah - que je définirai pour ma part comme le pôle névralgique de toute hétéronomie humaine qui ne s'est pas pliée au mythe aryen —, c'est se placer dans l'obligation de

4. Opération à laquelle s'est subtilement livrée l'Église de Rome. Voir, à ce propos, Georges-Élia Sarfati, Le Vatican et la Shoah, Paris, Berg international, 2000. 
comprendre la double illusion que constitue la «croyance» en la pureté du politique, mais aussi en la pertinence des sciences sociales. Après "Auschwitz», beaucoup ont douté de Dieu, souvent renié leur foi; mais que n'a-t-on d'abord douté du pouvoir de discernement et de la capacité prédictive des sciences sociales?

La définition du politique après "Auschwitz» ne peut donc pas s'accommoder de cette méconnaissance, et elle peut encore moins s'aboucher à la minimisation du "passé». La Shoah, appréhendée dans toute son extension historique et métahistorique, nous interdit d'envisager le sujet du politique à partir du postulat de l'humanisme ou de la philosophie classiques. Elle nous interdit aussi la confortable régression vers la prétendue recherche d'une autorité politique fondée sur la "prévention du risque». Une telle prévention n'existe pas, et ne saurait exister complètement, à l'échelle collective. Par contre, nous savons aujourd'hui que la peur de la peur est le meilleur antidote contre les libertés. La planétarisation de l'idéologie sécuritaire serait-elle l'indice manipulateur d'une planétarisation de cette peur de la barbarie et, subrepticement, le signe avant-coureur d'une barbarie fondée sur l'anesthésie des consciences? L'usage policier de cette prévention alternerait avec ses usages cathartiques (lesdites «politiques de la mémoire») pour ériger en mythe collectif les retombées symboliques du mythe nazi. Mais nous, pour qui la Shoah n'est le signe d'aucune altération de la barbarie, nous sommes trop attentifs au spectre de ce qu'Antelme appelle "la face cachée de l'homme» pour placer encore notre confiance dans la politique. Sous toutes les latitudes, la politique nous a montré son mépris de l'humain, et ce n'est pas la rhétorique consumériste qui nous fera douter de ses puissances de réification...

Le phénomène nazi nous a installés dans un monde surfait. Son œuvre: la Shoah - dont nous ne mesurons pas encore la portée transgénérationnelle - devrait donner la mesure d'une éthique réinventée. Une éthique ayant congédié dieux et maîtres, soucieuse d'arraisonner, c'est-à-dire de mettre à la raison, tout exercice du politique à l'aune d'une vigilance libertaire. Une éthique, en somme, inclinant de tout son poids vers la société civile, qui en émanerait en tant qu'expression contemporaine de la fonction critique. 